\title{
O USO DO CIM E A DIFUSÃO DAS IDEIAS NO CAMPO DAS POLÍTICAS PÚBLICAS NO SETOR DO GERENCIAMENTO DAS CIDADES ${ }^{1}$
}

\author{
THE USE OF CIM AND THE DIFUSION OF IDEAS IN THE FIELD OF \\ PUBLIC POLICIES IN THE SECTOR OF CITY MANAGEMENT
}

\author{
Ana Cláudia Rocha Cavalcanti \\ Universidade Federal de Pernambuco (UFPE) \\ rochacavalcanti@gmail.com \\ Flávio Antonio Miranda de Souza \\ Universidade Federal de Pernambuco (UFPE) \\ fdesouza67@gmail.com
}

\begin{abstract}
Resumo
A difusão de ideias no campo das políticas públicas tem sido amplamente investigada nas últimas décadas como forma de analisar como as políticas são formuladas; quem são os principais atores envolvidos (direta e indiretamente) e como as decisões para implementação de programas urbanos são realizadas. Nesse sentido, este artigo discute o tema da difusão abordando duas questões centrais: 1) A difusão de ideias na área de tecnologia da informação para a ações na escala urbana, examinando o caso do City Information Modelling (CIM) e, 2) O uso do CIM como ferramenta e os meios de difusão através da produção de políticas urbanas que buscam a melhoria da qualidade no gerenciamento das cidades. Para isto, o trabalho revisa de forma crítica a literatura internacional sobre difusão no campo da tecnologia da informação e no campo das políticas públicas. A relevância deste trabalho se dá pela busca da análise deste campo do conhecimento que cada dia mais aproxima a tecnologia de informação e o gerenciamento de cidades, mas que, para isto precisa passar pela esfera da produção, difusão e implementação de políticas públicas.
\end{abstract}

Palavras-chave: Difusão de políticas públicas. Tecnologia da informação. CIM.

\begin{abstract}
The diffusion of ideas in the field of public policy has been widely investigated in recent decades as a way to analyze how policies are made; who are the main actors involved (directly and indirectly) and how decisions for implementing urban programs are carried out. Therefore, this article discusses the topic of diffusion addressing two key questions: 1) The dissemination of ideas in the information technology area for actions in urban scale, examining the case of City Information Modelling (CIM) and, 2) The use CIM as a tool and means of delivery for the production of urban policies. Therefore, the paper critically reviews the international literature on diffusion in the field of information technology and in the field of public policy. The relevance of this work consists in the search for analysis of this field towards the production of knowledge that brings together information technology and management of cities, but in order to achieve this, they must go through the sphere of production, dissemination and implementation of public policies.
\end{abstract}

Keywords: Public policy diffusion. Information technology. CIM.

\footnotetext{
1 CAVALCANTI, A.; DE SOUZA, F. O uso do CIM e a difusão das ideias no campo das políticas públicas no setor do gerenciamento das cidades. In: ENCONTRO BRASILEIRO DE TECNOLOGIA DE INFORMAÇÃO E COMUNICAÇÃO NA CONSTRUÇÃO, 7., 2015, Recife. Anais... Porto Alegre: ANTAC, 2015.
} 


\section{INTRODUÇÃO}

No contexto da crescente utilização de dados espaciais integrados à semântica da forma para o uso de modelagem 3D da informação para a aplicação do gerenciamento com qualidade de cidades, um dos principais problemas que se pode observar seria a interoperabilidade (GROOT; McLAUGHLIN, 2000), de modo que um sistema universal teria que ser adotado pelos seus usuários. Para tanto, os fabricantes de programas computacionais têm disponibilizado no mercado diferentes programas, desde a criação de um SIG 3D a programas de modelagem da forma.

No campo da construção civil, costuma-se utilizar sistemas de gerenciamento de informação usando os conceitos do BIM, mas que também apresentam problemas de interoperabilidade. Modelos conceituais, sistemas e formatos de dados diversos dificultam a troca de plataformas em diferentes lugares (PAUWELS, 2014).

Uma vez que os sistemas de modelagem da informação tridimensional objetivam o gerenciamento desses dados de forma rápida, segura e eficiente, ainda há muito trabalho a ser realizado para integrar as diferentes formas de armazenar e manipular tais dados.

Inúmeras são as tentativas de padronizar o modus operandi desses sistemas visando o gerenciamento de informações e, este trabalho aponta que, nesse processo envolvem formas de difusão de ideias que afetam os meios pelos quais as informações são geradas e gerenciadas. Dessa forma, este trabalho consiste na análise sistemática acerca da difusão de ideias para compreender os limites e potencialidades desses modelos, assim como de suas adoções em modelos gerenciais de cidades.

Os dados disponíveis para o gerenciamento de cidades por meio da modelagem 3D podem estar armazenados em diferentes formas, além de conterem níveis de detalhamentos distintos, o que dificultam os usos visando a comparabilidade desses dados ao longo do tempo. Por outro lado, quando existentes, os dados de edificações mais recentes ou de regiões das cidades mais recentes podem apresentar modelos tridimensionais associados, enquanto que áreas das cidades mais antigas, muito comumente, não estão nem sequer representadas digitalmente. Por vezes, também, as áreas mais antigas não possuem bancos de dados associadas que poderiam ser integradas em modelos 3D.

Muitos são os problemas para a formação de um banco de dados e suas formas de atualizações. A modelagem 3D das edificações por si, já representa um entrave à sua completude enquanto ferramenta eficaz de planejamento e gestão devido à quase inexistências desses modelos. No que se refere ao gerenciamento das cidades, este cenário é ainda pior, pela quase inexistência de banco de dados dessa natureza nas cidades brasileiras. Mesmo assim, já se tem evidenciada a crescente demanda por gerenciamento de cidades com o uso de modelos digitais 3D. Em muitas vezes, a gestão de cidades, nos moldes tradicionais, já carece de dados pré-existentes, além da ausência de sistemáticas atualizações destes. Portanto, a tomada de decisão baseada na constante comparação de dados parece ser uma realidade ainda distante para grande parte das cidades brasileiras.

Os órgãos de planejamento e gestão de cidades dificilmente encontram-se estruturados com equipamentos que suportem bancos de dados extensos. As redes de Internet, quando existentes, nem sempre operam de forma constante e com qualidade. Os técnicos e suas repartições nem sempre estão interligados fisicamente, nem administrativamente. De fato, as realidades físico/institucional não correspondem às realidades de órgãos e sistemas equivalentes em países aonde os sistemas de gerenciamento de edificações, ou de cidades vêm sendo implementados. Mesmo assim, podem-se evidenciar canais de difusão dessas ideias que aos poucos criam demandas e justificam inovações.

A adoção de novas ideias e de ferramentas computacionais tem sido advogada em inúmeras formas e em diferentes países (RUSCHEL et al, 2013). Para que estas ocorram de 
forma universal há necessidade da difusão de inovações por meio de universidades, congressos acadêmicos, entre outros.

A difusão de inovações é bastante discutida no campo das inovações tecnológicas, assim como no campo das políticas públicas. Este trabalho consiste na exploração desses conceitos para ampliar nosso conhecimento sobre as implicações das inovações diante das crescentes demandas no uso de ferramentas computacionais para o gerenciamento com qualidade de cidades por meio do City Information Modelling (CIM).

Assim sendo, este artigo tem como objetivo discutir o tema da difusão (IKENBERY, 1990; ROGERS, 1995) abordando duas questões centrais: 1) A difusão de ideias na área de tecnologia da informação para a ações na escala urbana, examinando o caso do CIM e, como sua aplicação ainda não é em grande escala, 2) como isto está ocorrendo na prática no campo das políticas públicas para a gestão com qualidade de cidades.

\section{INFORMAÇÃO E QUALIDADE APLICADAS À MODELAGEM DO PRODUTO}

No campo das inovações tecnológicas no setor da informação para a construção civil, conceitos como Controle de Qualidade Total (Total Quality Control - TQC) são fundamentais para justificar inovações no campo gerencial, entre outros. Um dos princípios básicos da qualidade total consiste no constante aperfeiçoamento de processos sistêmicos para atingir eficiências cada vez mais primorosas (OSWALD; BURATI, 1992; FISHER et al, 1995).

Vale salientar que a qualidade é comumente relacionada ao mérito especial, excelência ou alto status. Entretanto, no campo da engenharia civil, de acordo com Ashford (2003) a qualidade envolve os conceitos de cumprimento de um requisito definido, de custobenefício, de adequação a um fim, ou ao conceito de satisfação do cliente. Dentro desses princípios que trataremos a qualidade na construção civil, e mais especialmente na cidade.

Primeiramente, com o objetivo de aprimoramento da qualidade e eficiência na construção civil, o BIM vem sendo amplamente estudado (EASTMAN et al, 2008; GRZYBEK et al, 2014) e difundido (ISO 29481-1; PAUWELS, 2014).

O BIM, fundamentado em princípios de linguagem baseados na modelagem orientadas para objetos, foi desenvolvido e vem sendo aperfeiçoado para capturar a semântica de objetos físicos, reais, e para compartilhar as informações desses produtos que visem solucionar problemas de uso e manutenção de edificações ao longo do tempo, entre os usuários de ferramentas e tecnologias gerenciais do tipo BIM (GRZYBEK et al, 2014).

As análises a respeito da implantação de sistemas que possibilitam o planejamento das cidades de forma tridimensional concluem que a aplicabilidade de tais sistemas é elevada, mesmo considerando os pontos negativos. Contudo, é necessário que os dados sejam coletados e armazenados de forma que sejam acessíveis e atualizados, ao mesmo tempo em que sejam comparáveis numa série histórica.

O City Information Modeling, mesmo não tendo simulações perfeitas, apresenta várias vantagens, pois trata-se de uma ferramenta que pode ser atualizada constantemente quanto às informações espaciais e físicas. Além disso, o sistema em 3D presente na modelagem $\mathrm{CIM}$ é mais preciso do que nos modelos $2 \mathrm{D}$ e contém diversas informações em um único programa.

Do ponto de vista da informação para tomada de decisão ligada ao campo do urbanismo, segundo Todor Stojanovski:

Urbanismo é uma mudança do plano para ação, o que relaciona Informação e teoria que pertencem à esfera científica, em contraste com a ação e regulação, que estão na esfera política. (STOJANOVSKI, 2013, p. 7). 
Nesse sentido, pode-se afirmar que o CIM diz respeito à esfera científica, mas serve como base para a esfera política.

\section{INOVAÇÃO DE IDEIAS}

As ideias são a matéria-prima que dão origem ao conhecimento (SOWELL, 1980), mas que, por existirem em abundância, tornam o processo de produção do conhecimento mais difícil, pois envolve escolhas e tomadas de decisões na seleção dessas, incluindo a classificação e autenticação das ideias.

Apesar de todos os problemas que estão associados à taxonomia, dependendo de quem classifica o quê, e como uma ideia é classificada, a classificação de algo pode não corresponder a uma realidade aproximada da coisa classificada. Assim sendo, Sowell (1980) aponta que as ideias podem ser classificadas quanto ao processo de autenticação destas, incluindo: 1) as que são sistematicamente preparadas para autenticação (teorias), 2) as que não derivam de nenhum processo sistemático (visões), 3 ) as que não sobrevivem a processos de autenticação (ilusões), 4) as que se eximem de processos de autenticações (mitos), 5) as que já passaram por processos de autenticação (fatos), e 6) as que não serão autenticadas (falsidades).

Os processos de autenticação das ideias, objetivando a produção do conhecimento, são variados e podem envolver procedimentos sistemáticos de verificação por meio de testes da estrutura lógica da teoria para sua consistência interna, ou a partir da observação de fatos no mundo real para testar sua consistência externa. Por outro lado, a autenticação das ideias pode partir de decisões baseadas no consenso, nas emoções ou nas tradições. $\mathrm{E}$ nestas bases pode significar a aprovação de um determinado grupo em um determinado tempo, ou ainda a aprovação de um determinado grupo que se vê como guardião de uma verdade particular, sendo tal grupo pertencente a uma elite que detém uma verdade que realmente interessa (SOWELL, 1980).

As ideias representam fatores que determinam as tomadas de decisão a partir da compreensão das ideias e da geração do conhecimento. As ideias são embutidas de valores determinados por indivíduos ou grupo de indivíduos, e ajudam a explicar o produto das políticas por serem determinantes importantes das políticas públicas. Entretanto, as ideias são filtradas e transformadas em conhecimento, que pode determinar as tomadas de decisão. As ideias por si não representam os determinantes das decisões, uma vez que estas estão relacionadas também com os interesses que motivam as pessoas ou instituições, sendo ambos relevantes para explicar as causas das tomadas de decisão. Nesse sentido, as ideias são consideradas como fator de geração do conhecimento para auxiliar na identificação de problemas que devem ser solucionados e que, para tanto, necessitam de ações de colaboração entre os atores para encontrarem uma solução (HAAS, 1990).

Nesse processo, há uma explicita ação de articulação entre os interesses dos atores envolvidos, que levam a atingir riscos mínimos para o sucesso. A concepção de que os indivíduos determinam suas decisões, baseadas numa perspectiva de maximização de seus ganhos, pode minimizar o papel das ideias (GOLDSTEIN; KEOHANE, 1993), uma vez que essas não seriam medidas. Além deste aspecto, há motivações econômicas para a produção e acumulação do conhecimento; uma vez que há uma minimização das necessidades na duplicação de produção de conhecimento que pode orientar a tomada de decisões e na difusão dessas ideias.

Neste sentido, a difusão de inovações de ideias parece fundamental para a compreensão do fenômeno estudado neste trabalho. Portanto, a próxima seção discute sobre a Teoria da Difusão para elucidar os conceitos necessários para seu melhor conhecimento. 


\section{DIFUSÃO DE INOVAÇÕES DE IDEIAS}

O conceito de difusão tem sido frequentemente analisado em perspectivas da difusão como um produto, ou a difusão como um processo (ELKINS; SIMMONS, 2005). No campo da abordagem da difusão como um produto, pode-se relacionar termos como isomorfismo (DIMAGGIO; POWELL, 1991), convergência (KERR, 1983; DREZNER, 2001), ondas (HUNTINGTON, 1991), entre outros. Por outro lado, no campo da difusão como processo, pode-se relacionar também o efeito demonstração (HUNTINGTON, 1991) imitação (JAKOBY, 2000), transferência de política (DOLOWITZ; MARSH, 2000), ou ainda efeito bandwagoning (IKENBERRY, 1990), entre outros.

Como apontado por Rogers (1995), a difusão refere-se ao processo pelo qual uma inovação é comunicada através de certos canais ao longo do tempo entre membros de um sistema social.

A comunicação é um processo pelo qual os participantes criam e compartilham informações para obter a compreensão comum sobre um determinado fenômeno, que detém características de novidade. Os participantes necessitam ser atualizados ao longo do tempo pelo acesso constante à informação, para assim reduzir as incertezas quanto às novidades dos acontecimentos.

Normalmente, uma nova ideia ou prática entra em uma comunidade a partir de uma fonte externa. Entretanto, a adoção da inovação acontece pelo contato interpessoal de uma rede de atores. $\mathrm{E}$ como pode ser esperado, há sempre aqueles que adotam a inovação assim que ela é introduzida, e há outros que apresentam certa resistência e aguardam para ver os resultados antes de decidir adotá-la. Ao longo do tempo, o número de pessoas que adotam a inovação aumenta, até que o público alvo em potencial, para a aceitação da ideia, é saturado (CAVALCANTI; De SOUZA, 2011).

Segundo a teoria da difusão das inovações, sua adoção passa por cinco estágios: conhecimento, persuasão, decisão, implementação e adoção. Isso seria explicado porque é necessário que haja conhecimento sobre a nova ideia (conhecimento). Para que isso ocorra, é necessária a adoção de programas de disseminação para ampliar o conhecimento sobre o fenômeno (persuasão). Uma vez que as pessoas passam a conhecer a nova ideia, intencionalmente tomam uma decisão quanto a experimentar ou não a inovação (decisão). $A$ partir de então, é necessária a aplicação da inovação (implementação). Caso a prática leve a resultados positivos, os indivíduos tendem a adotá-la em sua rotina (adoção). E em diversos estudos sobre difusão (ROGERS, 1995) esses estágios têm sido condensados em três: conhecimento, atitudes, e práticas. A adoção da difusão seria então influenciada pela forma como a inovação é percebida, assim como em função das características da organização e de seu público alvo.

As características das instituições que afetam a adoção de uma inovação incluem o grau de centralização de poder e controle da organização; a complexidade referente à capacidade técnica; a formalização dos processos por regras e procedimentos; a interconectividade dos sistemas de atores e suas redes, e a disponibilidade dos recursos presentes numa organização.

Ao longo do tempo, as instituições têm assumido papéis que levariam a duas formas de agir no processo de difusão: 1) a difusão das inovações aconteceria por mecanismos clássicos de coação (enforcement), tais como sanções, embargos, ou mesmo uso da força, e se fundamentam na tradição da economia política das teorias dos jogos e das ações coletivas (OLSON, 1971; AXELROD, 1984; PUTNAM, 1993), em que os atores se configurariam como racionais, que pesam os custos e benefícios de suas possíveis escolhas ao tomarem decisões em situações de coerção coletiva, e 2) a difusão aconteceria por mecanismos mais dinâmicos de negociações (management) entre os atores, que utilizam estratégias de 
resolução de problemas, interpretação de regras e transparência (HAAS, 1992; YOUNG, 1992) objetivando a capacitação dos atores e consequente aceitação das inovações, em vez de utilizarem ações visando à imposição daquelas pela coação de um grupo de atores sobre outros.

A Teoria da Difusão (ROGERS, 1995; IKENBERRY, 1990; HAAS, 1992; BERRY; BERRY, 1999) utiliza ferramentas apropriadas a lidar com questões numa escala macro. Sendo bastante apropriada para compreender como as relações entre os atores acontecem numa perspectiva entre instituições. Uma abordagem qualitativa, entretanto, é bastante apropriada para investigar relações numa microescala, para comparar e contrastar visões de mundo dos atores em ambas escalas - das instituições e dos atores individualmente; a partir da compreensão do fenômeno como ele acontece na escala micro.

A necessidade de uma linguagem comum entre os atores e instituições está muito bem apresentada por Rogers, uma vez que a relação entre os difusores da inovação e os receptores acontece mais apropriadamente quando esses comungam de "significados comuns, uma linguagem subcultural mútua, e são semelhantes em relação às características sociais e pessoais" (ROGERS, 1995, p. 19). Isto propiciaria um sistema social que favorece um ambiente para recepção de inovações, onde um certo grupo de indivíduos ou instituições serviriam de emissores ou transmissores de inovações; um outro grupo adotaria a inovação que foi transmitida através de canais de difusão que podem consistir de pessoas ou algum tipo de mídia que liga o transmissor ao que adota a inovação.

O difusionismo essencialista se baseia no princípio de que se uma determinada sociedade estivesse à frente de outras sociedades nas dimensões política, econômica e cultural, seria consensual a supremacia da sociedade mais desenvolvida sobre as outras, ainda em processo de desenvolvimento (BLAUT, 1977). Nesse sentido, os aspectos invisíveis da difusão, que fundamentam sua prática, fariam parte de um sistema de ideias, baseado em preconcepções de que as sociedades do Capitalismo avançado seriam o centro do sistema mundial. $O$ restante da sociedade mundial seria parte de uma sociedade periférica e estagnada, necessitando, portanto, das ideias centrais quanto à inovação e progresso.

Entretanto, algumas críticas a essa escola de pensamento já começam a ganhar corpo entre pesquisadores no campo da teoria social (MELO; COSTA, 1995; MELO, 2003, 2004) por considerarem que tanto os atores internacionais quanto os atores domésticos se organizam e se articulam no processo de difusão e assimilação de ideias.

\section{INOVAÇÕES DE IDEIAS E A GESTÃO DE PROJETOS PARA A CONSTRUÇÃO E PARA AS CIDADES}

No campo da gestão de projetos para a construção, cada vez mais necessita-se de um aperfeiçoamento gerencial que seja mais eficiente, com qualidade e inovativo. Frequentemente, nesse setor, lida-se com limitações de tempo para sua realização, mas que envolve o gerenciamento do ciclo de vida das edificações (HENDRICKSON, 2000). Um dos aspectos mais importantes nos resultados dos projetos que afetam seus ciclos de vida reside nas decisões adotadas no início de sua concepção, pois afetarão os custos de manutenção, a operação e funcionamento dos edifícios, etc. Nessa etapa, as decisões que aparentemente são de ordem arquitetônica, devem ser baseadas também em avaliações econômicas que afetarão o adequado financiamento das edificações, além do ambiente social e regulatório, das considerações tecnológicas, entre outras. Tais características envolvem habilidades de gestão que os arquitetos não costumam se apropriar, e isso tem comprometido os custos operacionais do ciclo de vida dos edifícios, e consequentemente, das cidades. 
No campo da gestão da construção dos edifícios, o uso de BIM é relativamente recente, portanto, a grande maioria das edificações existentes nas cidades não foi concebida nestes preceitos (El-MEKAWY, 2010). De fato, internacionalmente, muito pouco tem sido feito nesse processo na última década, e no Brasil, por ser ainda incipiente o seu uso, não se tem uma ideia de sua abrangência, mas pode-se arriscar afirmar que deve ser muito insignificante o número de projetos que sejam realizados por meio de sistema BIM.

No campo da educação, como relevante vetor de difusão de inovação de ideias (IKENBERRY, 1990; HAAS, 1992), este trabalho chama atenção para a relevância do tema e as disciplinas que podem ter integração. É importante pontuar que professores precisam tornar-se profissionais com visão integrada, compreendendo que um entendimento mais profundo de sua área de formação não é suficiente para o processo de ensino interdisciplinar. Faz-se necessário, portanto, apropriar-se também das múltiplas relações conceituais que sua área de formação estabelece com as outras ciências apontadas neste trabalho.

As cidades têm vivenciado um processo de crescimento sem procedentes nas últimas décadas e o volume de informações necessárias para sua gestão tem ficado cada vez maior. O grau de complexidade da interação desses dados também tem sido um dos principais entraves, principalmente no setor da modelagem tridimensional das cidades como uma alternativa para aperfeiçoar a gestão de dados para a tomada de decisões.

Os sistemas de modelagem de informação estão sendo muito requisitados, tais como o sistema usado para o City Information Modeling (CIM), uma vez que ele serve para gerenciar todos os tipos de infraestrutura incluindo pontes, estradas, túneis, prédios, entre outros. Além disso, esse sistema se configura como um jeito mais fácil de gerenciar projetos urbanos muito complexos de forma integrada e sustentável, uma vez que precisa da colaboração de vários setores e evita o desperdício. Por causa desses fatores, muitas construções já foram feitas utilizando essa ferramenta.

City Information Modelling é concebido e discutido como um sistema de blocos com relações dinâmicas ou conexões que definem e redefinem territórios. O CIM é análogo ao BIM (Building Information Modeling) só que em escala maior (STOJANOVSKI, 2013), pois engloba a cidade e é considerado uma evolução do Sistema de Informação Geográfica (SIG). O CIM usa o conceito de cidade relacionado ao espacial e ao das relações entre as pessoas e objetos, uma vez que, diferentemente de outros modelos, não é apenas um instrumento para ver os projetos, mas também apresenta uma série de dados associados ao sistema. Como existe uma gama de informações que pode ser acrescentada no programa, esse sistema divide a cidade em blocos que apresentam fronteiras, conexões e especificações internas que estão interligados. Do mesmo modo, existem vários tipos de conexões entre os blocos e essas subdivisões são chamadas de tags. Assim, a junção de todos esses blocos e conexões forma a cidade.

Logo, conclui-se que os sistemas de modelagem 3D são excelentes ferramentas para o planejamento e a construção das cidades, tanto na parte subterrânea quanto na parte acima do solo, sendo eficiente na visualização das obras e na junção dos dados. Porém, na representação em 3D torna-se necessária a descrição da composição da cidade, desde os limites da região e sua vegetação até os sistemas de água, transporte, estrada, (ZIURIENE et al, 2006), entre outros. A utilização de programas que possibilitem a visão espacial do relevo ou que comportem as informações necessárias é primordial no desenvolvimento desses projetos tridimensionais.

No entanto, além dos benefícios trazidos, existem alguns fatores negativos como o tempo necessário para o levantamento e cadastro dessas informações, assim como a necessidade de qualificação de mão de obra para o desenvolvimento do projeto. Dessa forma, investimentos no setor da formação profissional são necessários e buscam acompanhar os 
graus de evolução das inovações tecnológicas. Como os processos de inovação são cada vez mais frequentes e rápidos, há uma necessidade crescente de atualização profissional, criando demandas constantes, oriundas dessas inovações.

Por exemplo, o uso de ferramentas operando sistemas de informação para as cidades tem passado por significativos avanços por meio de Sistemas de Informações Geográficas que referenciam localizações por meio de coordenadas e agregam informações por meio de camadas de atributos, localizadas geograficamente. No caso do uso do CIM, o sistema possui sua própria forma de referenciar os espaços em blocos, agregando informações tridimensionais de modelos localizados em matrizes bidimensionais da cidade ou dos territórios (STOJANOVSKI, 2013). Muito ainda há de ser utilizado e experimentado para que se possa evoluir no conceito e no uso dessa ferramenta como forma de gerenciar cidades, tanto para auxiliar na tomada de decisões em situações previstas e imprevistas, como também para objetivar o controle urbano e a expansão dos serviços públicos.

\section{CONCLUSÕES}

A implantação de sistemas que possibilitam o planejamento das cidades de forma tridimensional pode ser bastante utilizada, pois o potencial da aplicabilidade de tais sistemas é elevado, mesmo considerando os pontos negativos acima relatados. Contudo, é necessário que os dados sejam coletados e armazenados de forma que sejam acessíveis, atualizados e possuam interoperabilidade em diferentes plataformas, ao mesmo tempo em que sejam comparáveis numa série histórica.

Os entraves administrativos, as limitações físicas e institucionais, além das infraestruturas deficientes das instituições ainda representam um grande e relevante entrave para a implantação de sistemas de gerenciamento de cidades por meio de modelagem da informação. Estes entraves não podem ser negligenciados.

Para se medir os impactos do uso do CIM como ferramenta em uma escala maior, a médio ou longo prazo, se faz necessário o seu conhecimento bem como os meios de sua difusão para os futuros profissionais no campo da construção civil, e da gestão de cidades e as reais implicações destes para processos de capacitação institucional oriundos das demandas geradas pelas inovações tecnológicas.

As inovações tecnológicas não podem ser advogadas sem hesitação, uma vez que demandam soluções básicas de adoção de sistemas que funcionem em diferentes plataformas.

O potencial de aperfeiçoamento nos sistemas de gerenciamento de cidades por meio da modelagem computacional 3D é inestimável, porém muito se faz necessário estudar e experimentar para aperfeiçoar esses modelos. Além disso, ainda se faz necessário realizar trabalhos integrados visando à interoperabilidade de ferramentas de manipulação da informação, tais como BIM e CIM.

Assim sendo, a aplicação dessas ferramentas pode alcançar um importante patamar no campo da construção civil e na gestão das cidades, ao promover interações teóricometodológicas que poderão aprimorar a gestão da informação cada vez mais associadas a produção do conhecimento por meio de novas tecnologias, em ambientes de estruturas corporativas que demandam trabalhos colaborativos e integrados. 


\section{AGRADECIMENTOS}

Os autores agradecem às alunas Maria Gabriela Specht e Maria Mirelle Reis (Programa Jovens Talentos - CAPES) e Maria Julia Lins.

\section{REFERÊNCIAS}

ASHFORD, J. The management of quality in construction. 4 ed. London: E \& F.N. Spon. 2003.

AXELROD, R., The Evolution of Cooperation, New York, Basic Books, 1984.

BERRY, F. and BERRY, W. Innovation and difusion models in policy process. In SABATIER, P. (Ed.) Theories of the policy process. Boulder and Oxford: Westview Press, 1999, pp. 169-200.

BLAUT, J. Two views of diffusion. Annals of the Association of American Geographers. No. 67, 1977, pp. 343-349.

CAVALCANTI, A. e DE SOUZA, F., Quando programas levam ao planejamento: difusão de ideias gerando conflitos na metrópole. Anais do Colóquio METROPOLES DAS AMÉRICAS: DESIGUALDADES, CONFLITOS E GOVERNANÇA, Montreal, 3 e 4 de outubro de 2011.

DIMAGGIO, P. and POWELL, P. The iron cage revisited: Institutional isomorphism collective rationality in organizational fields. In: POWELL, P. and DIMAGGIO, P. (Eds.) The New Institutionalism in organizational analysis. Chicago: Chicago University Press. 1991.

DOLOWITZ, D. P. and MARSH, D. Learning from abroad: the role of policy transfer in contemporary policy making. Governance, Vol. 13, No. 1, 2000, pp. 5-24.

DREZNER, D. Globalization and policy convergence. International Studies Review Vol.3, 2001, pp.55-78.

EASTMAN, C.; TEICHOLZ, P.; SACKS, R.; LISTON, K. BIM Handbook: A Guide to Building Information Modeling for Owners, Managers, Architects, Engineers, Contractors, and Fabricators; John Wiley \& Sons: Hoboken, NJ, USA, 2008.

ELKINS, Z. and SIMMONS, B. On waves, clusters, and diffusion: a conceptual framework. The Annals of the American Academy of Political and Social Science. Vol. 598, No. 1, 2005. pp. 33-51.

EI-MEKAWY, M. Integrating BIM and GIS for 3D city modelling The Case of IFC and City GML, Stokholm, Royal Institute of Technology (KTH). 2010.

FISHER, D.; MIERTSCHIN, S.; POLLOCK Jr., D. Benchmarking in construction industry. In Journal of Management in Engineering. N ${ }^{\circ}$ 11, pp. 50-57. 1995.

GOLDSTEIN, J. and KEOHANE, R. (Eds.) Ideas and foreign policy: beliefs, institutions and political change. Ithaca, Cornell Unviversity Press, 1993.

GROOT, R.,and McLAUGHLIN, J., Geospatial Data Infrastructure - Concepts, Cases, and Good Practice. Oxford: Oxford University Press. 2000.

GRZYBEK, H., XU, S., GULLIVER, S., FILLINGHAM, V. Considering the feasibility of Semantic Model Design in the built environment. Buildings, $N^{\circ} 4$, pp. 849879, 2014.

HAAS, E. When knowledge means power: three models of change in international organizations. Berkerley: University of California Press, 1990. 
HAAS, P. Introduction: epistemic communities and international policy coordination. International Organization. Vol. 46, No. 1, 1992, pp. 1-35.

HENDRICKSON, C. Project Management for Construction. Fundamental Concepts for Owners, Engineers, Architects and Builders, 2 ed. Carnegie Mellon University, Pittsburgh, 2000.

HUNTINGTON, S. The third wave: Democratization in the late twentieth century. Norman: University of Oklahoma Press, 1991.

IKENBERRY, G. J. The international spread of privatization policies: inducements, learning, and 'policy bandwagoning'. In: SULEIMAN, E. and WATERBURY, J. (Orgs.). The political economy of public sector. Boulder, Co.: Westview, 1990, pp. 88-110.

ISO 29481-1: Building Information Modelling-Information Delivery Manual-Part 1: Methodology and Format; International Organisation for Standardization: Geneva, Switzerland, 2010.

JAKOBY, W. Imitation and politics: Redesigning modern Germany. Ithaca: Cornell University Press, 2000.

KERR, N. L. Motivations losses in small groups: a social dilema analysis. Personality and Social Psychology. Vol. 45, 1983, pp. 819-828.

MELO, M. A. B. C. de, e COSTA, N. R. A difusão das reformas neoliberais: análise estratégica, atores, e agendas internacionais. Contexto Internacional. Vol. 17, No. 1, 1995, pp. 89-113.

MELO, M. A. B. C. de. Institutional choice and the diffusion of policy paradigms: Brazil and the second wave of pension reform. Artigo apresentado no IPSA Durban: International Congress, June, 2003. Mimeo.

MELO, M.A. B. C. de. Escolha institucional e a difusão dos paradigmas de política. In Dados. Rio de Janeiro, Vol. 47, No 1, 2004, pp. 169-206.

OLSON, M.. The Logic of Collective Action: Public Goods and the Theory of Groups, Harvard University Press, 1971.

OSWALD, T.; BURATI, J. Guidelines for implementing total quality management in the engineering and construction industry. Bureau of Engineering Research, University of Texas at Austin, 1992.

PAUWELS, P. Supporting decision-making in the building life-cycle using linked building data. Buildings, No 3, pp. 549-579, 2014.

PUTNAM, R. D. Diplomacy and domestic policy: the logic of two level games. In: EVANS, P., JACOBSON, H., and PUTNAM, R. Double edged diplomacy: international bargaining and domestic politics. University of California Press, 1993, pp. 431-467.

ROGERS, E. M. Diffusion of Innovations. New York: Free Press, 4th Edition, 1995.

SOWELL, T. Knowledge and decisions. New York: basic Books, 1980.

RUSCHEL, R. C.; ANDRADE, M.; MORAIS, M. O ensino de BIM no Brasil: onde estamos? Ambiente Construído, Porto Alegre, v. 13, n. 2, p. 151-165, abr./jun. 2013.

STOJANOVSKI, TODOR. City Information Modeling (CIM) and Urbanism: Blocks, Connections, Territories, People and Situations. ANAIS de SimAUD 13- Proceedings of the Symposium on Simulation for Architecture \& Urban Design, San Diego, 2013.

ZIURIENE, R. MESLIUTE, R. MAKUTENIENE, D. Development of 3D city model applying Cadastral Information. Geodesy and Cartography, Vol XXXII, No 2. 2006. 